\title{
The Lidose Hard Capsule Formulation of Fenofibrate is Suprabioavailable Compared to the Nanoparticle Tablet Formulation Under High-fat Fed Conditions
}

\author{
Roger K. Verbeeck ${ }^{1,2}$, Sophie De Niet ${ }^{3}$, Sonia Lebrun ${ }^{3}$, Mickael Tremege ${ }^{3}$, Tim W. Rennie ${ }^{2}$, Monte Coffiner ${ }^{3}$, Bruno \\ $\mathrm{Streel}^{4}$ and Bernard Cahay ${ }^{4}$ \\ 1 Louvain Drug Research Institute, Pharmacokinetics/drug Metabolism, Catholic University of Louvain, Brussels, \\ Belgium; ${ }^{2}$ School of Pharmacy, Faculty of Health Sciences, University of Namibia, Windhoek, Namibia; ${ }^{3}$ \\ Laboratoires SMB, Brussels, Belgium; ${ }^{4}$ Galephar MF, Marche-en-Famenne, Belgium.
}

Received, November 15, 2015; Revised, January 29, 2015; Accepted, January 30, 2015: Published January 31, 2015.

\begin{abstract}
Purpose: The therapeutic equivalence of multiple registered fenofibrate formulations, several of which are suprabioavailable and therefore marketed at lower dosage strengths than their reference products, is based on the results of bioequivalence studies. Most of these formulations show a higher bioavailability when taken with a high-fat meal. The relative bioavailability of two of these formulations, the $200 \mathrm{mg}$ Lidose hard capsules and the $145 \mathrm{mg}$ nanoparticle tablets, was assessed when taken with a high-fat meal. Methods: In this single dose, 2-way, randomized, crossover study, 24 healthy subjects received a 200 $\mathrm{mg}$ fenofibrate Lidose hard capsule (Test) and a $145 \mathrm{mg}$ nanoparticle tablet (Reference) under high-fat fed conditions. Plasma concentrations of fenofibric acid were measured up to 72 hours by using a validated LCMS/MS method. Results: The geometric mean ratios (Test/Reference) and the $90 \%$ confidence intervals for $\mathrm{AUC}_{0-\mathrm{t}}$ and $\mathrm{C}_{\max }$ were $1.37(131.58-142.88)$ and 1.38 (124.60 - 152.93), respectively. The median (range) $\mathrm{T}_{\max }$ values of fenofibric acid were $4.5 \mathrm{~h}(3.0-8.0 \mathrm{~h})$ and $3.25 \mathrm{~h}(1.0-6.5 \mathrm{~h})$ after administration of the Lidose hard capsule and the nanoparticle tablet, respectively. Conclusion: Under high-fat fed conditions the extent of fenofibrate absorption was $37 \%$ higher for the $200 \mathrm{mg}$ Lidose hard capsule compared to the 145 mg nanoparticle tablet, which is exactly as expected based on a mg-to-mg weight basis. The results of the present study underline the importance of assessing bioequivalence of fenofibrate formulations under identical fed conditions, and preferentially after a high-fat meal as this condition represents the worst-case scenario. Furthermore, the results of this study demonstrate that the $145 \mathrm{mg}$ nanoparticle tablet is not bioequivalent to the $200 \mathrm{mg}$ Lidose hard capsule when administered under high-fat meal conditions.
\end{abstract}

This article is open to POST-PUBLICATION REVIEW. Registered readers (see "For Readers") may comment by clicking on ABSTRACT on the issue's contents page.

\section{INTRODUCTION}

Fenofibrate is indicated for use in the treatment of primary hypercholesterolemia, mixed dyslipidemia and hypertriglyceridemia in adults who have not responded to non-pharmacological measures. Its lipid-modifying effects are mediated by activation of peroxisome proliferator-activated receptor- $\alpha$ (1,2). The European Commission (European Referral) also endorsed in 2010 an additional indication for fenofibrate, i.e. mixed hyperlipedemia in patients at high cardiovascular risk in addition to a statin when triglycerides and HDL- cholesterol are not adequately controlled. Fenofibrate was the only fibrate to receive this indication based on the results of the ACCORDLIPID study (3). Fenofibrate is the isopropyl ester of fenofibric acid (4). It is a prodrug which, following oral administration, is rapidly hydrolyzed by esterases to its active metabolite, fenofibric acid. Unmetabolized fenofibrate has been reported to be undetectable in plasma samples following oral administration (5).

Fenofibrate has been marketed since 1975 $(6,7)$. It is a substance with poor water solubility and the original formulation was a $100 \mathrm{mg}$ tablet to be taken as 3 tablets at the same time, because fenofibrate had a poor oral bioavailability. It was also recommended to be taken with food to increase the extent of absorption (7). A $200 \mathrm{mg}$ capsule formulation of micronized fenofibrate was subsequently developed as well as a $160 \mathrm{mg}$ micronized fenofibrate microcoated tablet.

Corresponding Author: Roger K Verbeeck; Louvain Drug Research Institue, Catholic University of Louvain, Brussels, Belgium; Email: roger.verbeeck@uclouvain.be 
By using smaller fenofibrate particle sizes these newer formulations showed improved bioavailability (suprabioavailability) and were registered at lower dose strengths compared to the initial formulations (7). SMB-Galephar (Brussels, Belgium) developed a $200 \mathrm{mg}$ hard capsule formulation with a semi-solid content (Lidose, patent EP 0801562B1) into which fenofibrate is homogeneously dispersed within a lipid excipient mixture (7). This product was considered to be bioequivalent to the $200 \mathrm{mg}$ micronized fenofibrate reference product on the basis of a crossover relative bioavailability study (8). A major drawback of all these fenofibrate formulations is that adequate oral absorption requires their administration with a high-fat meal (9-11). More recently, a new nanoparticle tablet formulation of fenofibrate has become available in $48 \mathrm{mg}$ and $145 \mathrm{mg}$ dose strengths $(12,13)$. Unlike previous fenofibrate formulations, the oral bioavailability of this nanoparticle formulation was apparently not affected by food and, consequently, it has been recommended that this nanoparticle formulation can be administered without regard to food intake $(12,13)$.

Differences in bioavailability of the various fenofibrate formulations mean that they are not equivalent on a milligram-to-milligram basis. Consequently, marketed oral fenofibrate formulations may not only differ substantially in available dose strengths, but also in the requirement to be taken with a meal (9). The present bioequivalence study was undertaken to investigate the bioavailability of a $145 \mathrm{mg}$ fenofibrate nanoparticle tablet relative to the 200 mg fenofibrate Lidose hard capsule when administered to healthy volunteers under high-fat conditions.

\section{METHODS}

\section{Subjects and study protocol}

The study protocol was approved by the Ethics Committee of the University of Medicine and Pharmacy "Iuliu Hatieganu", Cluj-Napoca, Romania. The study was performed in accordance with the version of the Helsinki Declaration in force at the time of the study and Good Clinical Practice Guidelines. The clinical part of the study was conducted by S.C. Kynetyx HT S.R.L., ClujNapoca, Romania. Twenty-four healthy nonsmoking subjects (12 males and 12 females) between the age of 18 and 36 years $(24.7 \pm 4.7$ years, mean $\pm \mathrm{SD}$ ), having a body mass index between 18.8 and $27 \mathrm{~kg} / \mathrm{m}^{2}\left(22.3 \pm 2.6 \mathrm{~kg} / \mathrm{m}^{2}\right)$ were included after having undergone a complete medical examination (clinical observation, ECG, haematological, biochemical, and serological screening). Written informed consent was obtained for every subject prior to enrolment.

\section{Study design}

The study was designed as a single dose, open label, two-period, two-treatment, two-sequence, crossover study to compare the bioavailability of two oral fenofibrate formulations: Fenogal Lidose hard gelatin capsules containing $200 \mathrm{mg}$ fenofibrate (Laboratoires SMB, Brussels, Belgium) versus LipanthylNano film-coated tablets containing $145 \mathrm{mg}$ fenofibrate as nanoparticles (Abbott Products, Belgium). The two study periods were separated by a washout of minimum 8 days. An equal number of subjects was randomly assigned to each of the two possible administration sequences. During each study period, subjects were admitted to the clinical facility in the evening prior to the administration. One $200 \mathrm{mg}$ fenofibrate Lidose hard gelatin capsule (Test) or one $145 \mathrm{mg}$ fenofibrate nanoparticle tablet (Reference) was administered with $240 \mathrm{ml}$ of water 30 minutes after the start of a high fat, high calorie breakfast. Water was allowed ad libitum except for one hour before and one hour after drug administration. Standard meals were given at $4 \mathrm{~h}$ (lunch), $8 \mathrm{~h}$ (snack) and $13 \mathrm{~h}$ (dinner) following dosing. Study subjects were confined to the clinical center from the evening before until 24 hours after dosing. A total of twenty-two 6-ml blood samples were collected per period: pre-dose $(-0.35 \mathrm{~h})$, and 1.0 , $2.0,2.5,3.0,3.5,4.0,4.25,4.5,4.75,5.0,5.25$, $5.5,5.75,6.0,6.5,7.0,8.0,12,24,48$ and 72 hours following administration. Subjects were monitored for adverse events throughout the course of the study until 30 days

\section{Drug Analysis}

Fenofibric acid plasma concentrations were determined by liquid chromatography coupled to a tandem mass spectrometry detection system (LC-MS/MS, API 3000, AB Sciex, Toronto, Canada). In short, to $40 \mu 1$ of plasma were added $460 \mu \mathrm{l}$ of methanol, containing deuterated fenofibric acid (d6-fenofibric acid) as internal standard. An aliquot of the supernatant was injected onto an end-capped dodecylsilica column (Synergi Max-RP, $80 \AA$ pore size, $4 \mu \mathrm{m}$ particle size, $150 \mathrm{~mm} \mathrm{x} 2 \mathrm{~mm}$ internal diameter, Phenomenex). The mobile phase, consisting of a mixture of acetonitrile, methanol and an aqueous 
solution of $5 \mathrm{mM}$ ammonium acetate adjusted to $\mathrm{pH}$ of 4.5 with acetic acid $(37.5: 37.5: 25, \mathrm{v} / \mathrm{v} / \mathrm{v})$, was delivered to the column at a flow rate of 0.2 $\mathrm{ml} / \mathrm{min}$. Mass spectrometric detection was carried out using an Applied Biosystems API 4000 Triple Quadrupole instrument (Thornhille, Toronto, Canada) equipped with a TurbulinSpray interface. The MS/MS ion transitions monitored were $\mathrm{m} / \mathrm{z}$ $317.1 \rightarrow 230.8$, and $323.1 \rightarrow 230.7$ for fenofibric acid and d6-fenofibric acid, respectively. The lower limit of quantification of fenofibric acid in plasma was $0.2502 \mu \mathrm{g} / \mathrm{ml}$. Details of the analytical method are described elsewhere (12). The bioanalytical method was validated regarding selectivity, accuracy, precision, stability, backconversion and matrix effect to comply with the EMA guidelines (11). Incurred sample reanalysis showed that $97.9 \%$ of the reanalysed samples were within $20 \%$ of the mean.

\section{Pharmacokinetic analysis}

Pharmacokinetic parameters of fenofibric acid were obtained by non-compartmental analysis. $\mathrm{C}_{\max }$, the maximum plasma concentration, and $\mathrm{T}_{\max }$, the time to reach $\mathrm{C}_{\max }$, were obtained directly from the observed fenofibric acid plasma concentration-time data. $\mathrm{AUC}_{0-\mathrm{t}}$ (area under the plasma concentration-time curve from time 0 to the time of the last quantifiable plasma concentration) was calculated by using the mixed linear-log trapezoidal method (Kinetica ${ }^{\circledR}$, version 5.0, Thermo Fisher Scientific). Total area under the curve $\left(\mathrm{AUC}_{0-\infty}\right)$ was obtained as the sum of $\mathrm{AUC}_{0-\mathrm{t}}$ and the extrapolated area under the curve $\left(\mathrm{AUC}_{\mathrm{t}-\infty}\right)$, which was calculated by dividing the fitted last quantifiable plasma concentration by $0.693 / t^{1} / 2$, and $t^{1} / 2$ (the terminal plasma half-life of fenofibric acid) was estimated from the slope of the terminal phase of the semi-logarithmic plasma concentration versus time curve.

\section{STATISTICAL ANALYSIS}

Statistical evaluations of $\mathrm{AUC}_{0-\mathrm{t}}, \mathrm{AUC}_{0-\infty}$ and $\mathrm{C}_{\max }$ were performed after $\ln$-transformation using ANOVA for a two-way crossover design. After the 4 dropouts the sequence for the remaining 20 subjects was still balanced (21). The general linear model (GLM) procedure was used to assess the effect of sequence, subject nested within sequence, period and formulation on these $\mathrm{BE}$ metrics (Kinetica ${ }^{\circledR}$, version 5.0, Thermo Fisher Scientific and EquivTest/PK ${ }^{\circledR}$, Statistical Solutions, 2006, gave the same results). For the assessment of bioequivalence, the $90 \%$ confidence intervals were calculated around the geometric mean ratio Test/Reference according to the two one-sided tests procedure of Schuirmann (14). Bioequivalence was concluded when the $90 \%$ confidence interval for the primary bioequivalence metrics $\mathrm{AUC}_{0-\mathrm{t}}$ and $\mathrm{C}_{\max }$ fell within the $80-125 \%$ acceptance limits (15). $\mathrm{T}_{\max }$ is not considered a primary metric for bioequivalence assessment, but it was nevertheless analysed by the non-parametric Friedman test. Descriptive results are expressed as mean \pm standard deviation for continuous variables (e.g. $\mathrm{C}_{\max }, \mathrm{AUC}$ ) and median (range) for the discrete variable $\mathrm{T}_{\max }$.

\section{Safety assessment}

Vital signs (blood pressure, heart rate) were recorded for each subject at screening, throughout the confinement periods and at the end of the study. Laboratory safety parameters (urinalysis, hematology, clinical chemistry) were measured at screening and at the end of the study. All study subjects were monitored for adverse events throughout the entire confinement periods and until 30 days after the administration of the last dose.

\section{RESULTS}

After screening, 24 subjects were randomised to receive a single dose of the test and reference fenofibrate formulations. One subject withdrew before the start of the study, another subject dropped out during period I, and 2 subjects withdrew from the study during period II. As a result, the pharmacokinetic and statistical analyses are based on the results of the 20 study subjects who completed both phases of the study. The mean fenofibric acid plasma concentrationtime profiles following administration of a single dose of the test $(\mathrm{T})$ and reference $(\mathrm{R})$ prepration are shown in figure 1. Pharmacokinetic parameter estimates of fenofibric acid are summarized in Table 1. Peak plasma concentrations of fenofibric acid were reached at $4.5 \mathrm{~h}(3.0 \mathrm{~h}-8.0 \mathrm{~h})$ [median (range)] following administration of test formulation, and at $3.25 \mathrm{~h}(1.0 \mathrm{~h}-6.5 \mathrm{~h})$ following administration of reference formulation. The difference in $\mathrm{T}_{\max }$ between Test and Reference formulation was not statistically significant (Friedman test). The plasma half-life of fenofibric acid was $18.6 \mathrm{~h}$ (range: $7.3-28.6 \mathrm{~h}$ ) and $18.7 \mathrm{~h}$ (range: $6.4-28.4 \mathrm{~h}$ ) for Test and Reference formulation, respectively. On average 


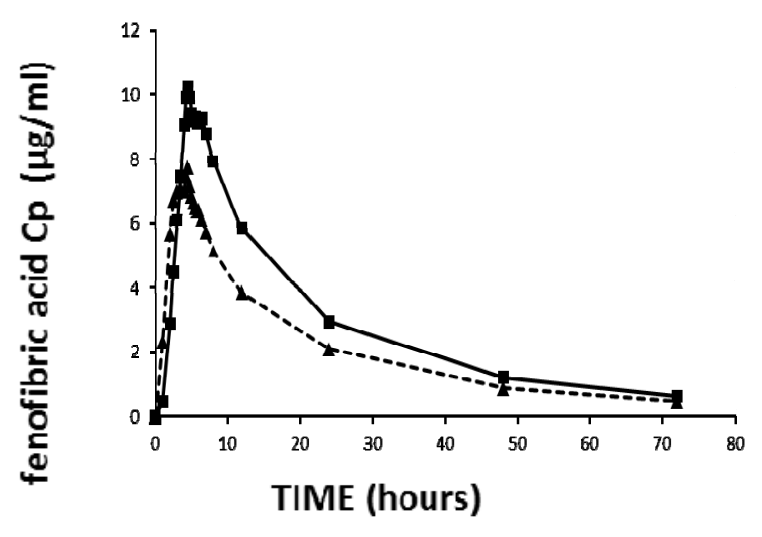

Figure 1. Mean fenofibric acid plasma concentration-time profiles in 20 healthy subjects after single dose administration of a $200 \mathrm{mg}$ fenofibrate Lidose hard capsule (preparation) and a $145 \mathrm{mg}$ fenofibrate nanoparticle tablet $(\mathbf{A}-\cdots)$ Reference formulation).

$8.0 \pm 3.5 \%$ (Test formulation) and $8.6 \pm 3.4 \%$ (Reference formulation) of the total area under the curve $\left(\mathrm{AUC}_{0-\infty}\right)$ was located in the extrapolated part (i.e. $\mathrm{AUC}_{\mathrm{t}-\mathrm{o}}$ ). The geometric mean ratios $(\mathrm{T} / \mathrm{R})$ and $90 \%$ confidence intervals for $\mathrm{C}_{\max }$, $\mathrm{AUC}_{0-\mathrm{t}}$ and $\mathrm{AUC}_{0-\infty}$ are summarized in Table 2. The intra-individual variability $\left(\mathrm{CV}_{\text {anova }}\right)$ of $\mathrm{C}_{\max }$ was $18.9 \%$, and was smaller for $\mathrm{AUC}_{0-\mathrm{t}}(7.5 \%)$ and $\mathrm{AUC}_{0-\infty}(7.9 \%)$ (Table 2).

A total of 8 treatment emergent adverse events were reported by 7 subjects in the study. Six of them were reported with the $145 \mathrm{mg}$ nanoparticle tablets, and 2 were reported with the $200 \mathrm{mg}$ Lidose hard capsules. Five adverse events were of mild intensity and 3 were of moderate intensity. Four adverse events (all reported with the $145 \mathrm{mg}$ nanoparticle tablets) were considered related with the study drug. The most common treatment-related adverse event was headache. No serious adverse events occurred during the study.

\section{DISCUSSION}

Fenofibrate was first introduced in 1975 but the oral bioavailability of this standard formulation was very low especially when taken without a high-fat meal $(6,9)$. Micronized fenofibrate formulations were subsequently developed to increase the water solubility and hence the oral bioavailability of fenofibrate (2). A micronized fenofibrate capsule formulation was introduced in Europe in 1992, but was later replaced by a micronized microcoated tablet with a $25 \%$ increase in bioavailability compared to the micronized capsule formulation. The Lidose drug delivery technology, i.e. a hard capsule with a semi-solid content into which fenofibrate is homogeneously dispersed within a lipid excipient mixture, was also used in an attempt to improve the oral fenofibrate bioavailability (9). Despite improvement in oral bioavailability of the micronized fenofibrate tablets and Lidose fenofibrate hard capsules, these formulations still require the presence of food, particularly a highfat meal, to maximize the extent of fenofibrate absorption (2,9). More recently, fenofibrate formulations based on nanoparticle technology were developed in an attempt to reduce or completely eliminate the food effect $(1,9)$. Ling et al. has published a detailed description of the currently available fenofibrate and fenofibric acid formulations and the effect of food on their oral bioavailability (9).

\begin{tabular}{|c|c|c|}
\hline Parameter & $\begin{array}{l}\text { TEST } \\
200 \mathrm{mg} \text { Lidose capsules }\end{array}$ & $\begin{array}{l}\text { REFERENCE } \\
145 \text { mg nanoparticle tablets }\end{array}$ \\
\hline $\mathrm{T}_{\max }, \mathrm{h}$ & $4.5(3.0-8.0)$ & $3.25(1.0-6.5)$ \\
\hline $\mathrm{C}_{\max }, \mu \mathrm{g} / \mathrm{ml}$ & $12.6 \pm 2.9$ & $9.2 \pm 2.3$ \\
\hline $\mathrm{AUC}_{0-\mathrm{t}}, \mu \mathrm{g} \cdot \mathrm{h} / \mathrm{ml}$ & $193.3 \pm 84.9$ & $138.8 \pm 51.3$ \\
\hline $\mathrm{AUC}_{0-\infty}, \mu \mathrm{g} \cdot \mathrm{h} / \mathrm{ml}$ & $211.9 \pm 101.0$ & $153.2 \pm 63.4$ \\
\hline $\mathrm{t}^{1 / 2}, \mathrm{~h}$ & $18.8 \pm 5.8$ & $18.8 \pm 5.6$ \\
\hline
\end{tabular}


Table 2: Geometric mean ratios (GMR) T/R, 90\% confidence intervals ( $90 \% \mathrm{CI}$ ) and intrasubject variabilities $\left(\mathrm{CV}_{\text {anova }}\right)$ for $\mathrm{C}_{\max }, \mathrm{AUC}_{0-\mathrm{t}}$ and $\mathrm{AUC}_{0-\infty}$ following single dose administration of Test $(200 \mathrm{mg}$ Lidose hard capsules) and Reference formulation ( $145 \mathrm{mg}$ nanoparticle tablets) to 20 healthy subjects.

\begin{tabular}{llll}
\hline BE parameter & $\boldsymbol{G M R}$ & $\mathbf{9 0 \%} \boldsymbol{C I}$ & $\boldsymbol{C V}_{\text {anova }}(\%)$ \\
$\mathrm{C}_{\max }, \mu \mathrm{g} / \mathrm{ml}$ & 1.38 & $124.60-152.93$ & 18.9 \\
$\mathrm{AUC}_{0-\mathrm{t}}, \mathrm{g} . \mathrm{h} / \mathrm{ml}$ & 1.37 & $131.58-142.88$ & 7.5 \\
$\mathrm{AUC}_{0-\infty}, \mu \mathrm{g} . \mathrm{h} / \mathrm{ml}$ & 1.36 & $130.38-142.08$ & 7.9 \\
\hline
\end{tabular}

During the development of these different formulations to optimize the extent of fenofibrate absorption and to suppress the food effect, bioequivalence studies were carried out to show therapeutic equivalence between the marketed reference formulation and the newly developed, often suprabioavailable, formulations. Unfortunately, these bioequivalence studies were not always carried out under the same fed condition. For example, the $48 \mathrm{mg}$ and $145 \mathrm{mg}$ fenofibrate nanoparticle tablets were shown to be bioequivalent to the $200 \mathrm{mg}$ micronised fenofibrate capsule under low-fat conditions (18). However, for both the $48 \mathrm{mg}$ and $145 \mathrm{mg}$ nanoparticle tablets, the $\mathrm{AUC}_{0-\mathrm{t}}$ of fenofibric acid was on average $13 \%$ lower compared to the 200 micronized capsule formulation. Bioequivalence could still be demonstrated because the intraindividual variability of the AUC for fenofibric acid was low and a large number of subjects was used (72 subjects were enrolled and 68 completed the study) (18). Since the extent of the food effect may be affected by the fat content of the meal, the unanswered question is whether under high-fat fed conditions the $145 \mathrm{mg}$ nanoparticle tablet would still have been bioequivalent to the $200 \mathrm{mg}$ micronized capsule. On the other hand, in a randomized, 3-way, food-effect study on the 145 mg nanoparticle tablet, it was shown that the relative bioavailability following administration with a low-fat and a high-fat meal was very similar to that after administration of the nanoparticle formulation on an empty stomach $(12,18)$.

When the bioavailability of a $67 \mathrm{mg}$ and 200 $\mathrm{mg}$ Lidose hard capsules was compared to that of the $67 \mathrm{mg}$ and $200 \mathrm{mg}$ micronized fenofibrate capsules, the Lidose $67 \mathrm{mg}$ and $200 \mathrm{mg}$ formulations were shown to be bioequivalent to the $67 \mathrm{mg}$ and $200 \mathrm{mg}$ micronized fenofibrate capsules, respectively, under high-fat fed conditions (8). In the bioequivalence study described in this publication, the bioavailability of a $145 \mathrm{mg}$ fenofibrate nanoparticle tablet was compared to that of a $200 \mathrm{mg}$ fenofibrate Lidose hard capsule under high-fat fed conditions. Based on the results of the above described bioequivalence studies $(8,18)$ in which both the $145 \mathrm{mg}$ nanoparticle tablets and the $200 \mathrm{mg}$ Lidose formulations were demonstrated to be bioequivalent to the same reference i.e. a $200 \mathrm{mg}$ fenofibrate micronized capsule, but in different studies, one would expect the $200 \mathrm{mg}$ Lidose capsules to be bioequivalent to the $145 \mathrm{mg}$ nanoparticle Lipanthyl ${ }^{\circledR}$ tablets. However, the results of the present study showed a $37 \%$ higher extent of fenofibrate absorption for the $200 \mathrm{mg}$ Lidose capsules compared to the $145 \mathrm{mg}$ nanoparticle tablets. In fact the $200 \mathrm{mg}$ Lidose formulation showed an equivalent AUC on a weight-to-weight basis compared to the $145 \mathrm{mg}$ nanoparticle formulation.

During the last 20 years several reformulations of the marketed branded fenofibrate preparations have been developed as well as generics of out of patent fenofibrate preparations. Registration of these reformulated and generic fenofibrate preparations was based mainly on bioequivalence studies to show therapeutic equivalence between the reformulated or generic preparation and a marketed reference formulation. In a recent paper by Downing et al., the authors show how Abbott Laboratories, the maker of branded fenofibrate, has produced several suprabioavailable reformulations in a successful attempt to maintain a dominant market share by preventing generic competition (19). This has resulted in a situation where many different fenofibrate formulations are on the market, not necessarily possessing the same dose strengths because some are suprabioavailable compared to others $(9,19)$. In addition, for some of these fenofibrate formulations it is claimed that there is no food effect, although the various bioequivalence studies have certainly not been carried out under the same fed conditions. The EMA and the FDA recommend to use a highcalorie, high-fat meal during food effect bioavailability and bioequivalence studies because such meal is expected to provide the greatest effects on gastrointestinal physiology and hence on the bioavailability characteristics of the test formulation $(15,20)$. In addition, for fenofibrate administration the situation is 
complicated by the fact that the patients are recommended to adhere to low-fat dietary guidelines. It is clear that the food and fat effects seen with most, if not all, currently marketed fenofibrate formulations may lead to unpredictable, inconsistent and non-optimal bioavailability especially when switching patients from one fenofibrate formulation to another. Consequently, in our opinion it would be necessary to systematically carry out the bioequivalence studies on fenofibrate formulations under high-fat fed conditions, which represent the "worst-case scenario".

In conclusion, the results of this bioequivalence study show that, under high-fat fed conditions, the $200 \mathrm{mg}$ fenofibrate Lidose hard capsules are suprabioavailable (the GMR for AUC is 1.37) compared to the $145 \mathrm{mg}$ fenofibrate nanoparticle tablet. It would be interesting to assess how this difference in fenofibric acid exposure affects the patient's triglyceride levels.

\section{ACKNOWLEDGEMENTS}

RKV and TWR have no conflict of interest. SDN, SL, MT, MC, BS and BC are employees of Laboratoires SMB, the company that developed the Fenogal Lidose hard capsules.

\section{REFERENCES}

1. Keating GM and Croom KF: Fenofibrate - a review of its use in primary dyslipidaemia, the metabolic syndrome and Type 2 diabetes mellitus. Drugs 2007; 67: 121-153.

2. Keating GM and Ormrod D: Micronised fenofibrate - an updated review of its clinical efficacy in the management of dyslipidemia. Drugs 2002; 62: 1909-1944.

3. EMA/CHMP/580013/2012: Assessment report for fenofibrate, bezafibrate,ciprofibrate and gemfibrozil medicinal products pursuant to Article 31 of Directive 2001/83/EC, as amended (Procedure number: EMEA/H/A-1238), February 2011.

4. Balendiran GK, Rath N, Kotheimer A, Miller C, Zeller M and Rath NP: Biomolecular chemistry of isopropyl fibrates. J. Pharm. Sci. 2012; 101: 1555-1569.

5. Streel B, Hubert $P$ and Ceccato A: Determination of fenofibric acid in human plasma using automated solid-phase extraction coupled to liquid chromatography. J. Chromatogr. B Biomed. Sci. Appl. 2000; 742: 391-400.

6. Balfour JA, McTavish D and Heel RC: Fenofibrate - A review of its pharmacodynamic and pharmacokinetic properties and therapeutic use in dyslipidaemia. Drugs 1990; 40; 260-290.
7. Guichard JP, Blouquin $\mathrm{P}$ and Qing Y: A new formulation of fenofibrate: suprabioavailable tablets. Curr. Med. Res. Opin. 2000; 16: 134-138.

8. Sonet B, Vanderbist F, Streel B and Houin G: Randomised crossover studies of the bioequivalence of two fenofibrate formulations after administration of a single oral dose in healthy volunteers. Arzneim.-Forsch./Drug Res. 2002; 52: 200-204.

9. Ling H, Luoma JT and Hilleman D: A review of currently available fenofibrate and fenofibric acid formulations. Cardiol. Res. 2013; 4: 47-55.

10. Najib J: Fenofibraae in the treatment of dislipidemia - a review of the data as they relate to the new suprabioavailable tablet formulation. Clin. Ther. 24: 2022-2050, 2002.

11. Guivarc'h P-H, Vachon MG and Fordyce D: A new fenofibrate formulation: results of six singledose, clinical studies of bioavailability under fed and fasting conditions. Clin. Ther. 2004; 20: 1456-1469.

12. Sauron R, Wilkins M, Jessent V, Dubois A, Maillot $\mathrm{C}$ and Weil A: Absence of a food effect with a $145 \mathrm{mg}$ nanoparticle fenofibrate tablet formulation. Int. J. Clin. Pharmacol. Ther. 2006; 44: 64-70.

13. Lipanti ${ }^{\circledR}$ Supra $145 \mathrm{mg}$ film-coated tablets, Summary of Product Characteristics, Abbott Laboratories Ireland Ltd., Dublin, Ireland, 22/03/2011.

14. Mertens B, Cahay B, Klinkenberg R and Streel B: An automated method for the simultaneous determination of pravastatin, 3-hydroxy isomeric metabolite, pravalactone and fenofibric acid in human plasma by sensitive liquid chromatography combined with diode array and tandem mass spectrometry detection. J. Chromatogr. A 2008; 1189: 493-502.

15. EMA Guideline on bioanalytical method validation, EMEA/CHMP/EWP/192217/2009, 21 July 2011

(http://www.ema.europa/docs/en_GB/document library/Scientific_guideline/2011/08/WC5001096 86.pdf).

16. Schuirmann DJ: A comparison of the two onesided tests procedure and the power approach for assessing the equivalence of average bioavailability. J. Pharmacokinet. Biopharm. 1987; 15: 657-680.

17. EMA Guideline on the investigation of bioequivalence, CPMP/EWP/QWP/1401/98 Rev. 1/Corr **, London, 20 January, 2010 (http://www.ema.europa.eu/docs/en_GB/docume nt_library/Scientific_guideline/2010/01/WC5000 70039.pdf.

18. Clinical Pharmacology and Biopharmaceutics Review(s), Application number 21-656, CDER, August 4.2004

(http://www.accessdata.fda.gov/drugsatfda_docs/ nda/2004/021656s000_Tricor_BioPharmR.pdf. 
19. Downing NS, Ross JS, Jackevicius CA and Krumholz HM: Avoidance of generic competition by Abbott Laboratories' fenofibrate franchise. Arch. Inter. Med. 2012; 172: 724-730.

20. FDA Guidance for Industry: Food-effect bioavailability and fed bioequivalence studies, December 2002 (http://www.fda.gov/downloads/regulatoryinform ation/guidances/ucm126833.pdf).

21. Schütz H, Labes D and Fuglsang A: Reference data sets for 2-treatment, 2- sequence, 2-period bioequivalence studies. AAPS J, Sep 12, Epub ahead of print, 2014. 